\title{
Commentary: Promising future solution for unmet needs in lung transplantation
}

\author{
Toyofumi F. Chen-Yoshikawa, MD
}

\author{
From the Department of Thoracic Surgery, Kyoto University Graduate School of Medicine, Kyoto, Japan. \\ Disclosures: Author has nothing to disclose with regard to commercial support. \\ Received for publication Dec 18, 2018; accepted for publication Dec 18, 2018; available ahead of print Jan 29, \\ 2019. \\ Address for reprints: Toyofumi F. Chen-Yoshikawa, MD, Department of Thoracic Surgery, Kyoto University \\ Graduate School of Medicine, 54 Shogoin Kawahara-cho, Sakyo-ku, Kyoto 606-8507, Japan (E-mail: \\ tfchenyoshikawa@gmail.com). \\ J Thorac Cardiovasc Surg 2019;157:2107-8 \\ $0022-5223 / \$ 36.00$ \\ Copyright (c) 2018 by The American Association for Thoracic Surgery \\ https://doi.org/10.1016/j.jtcvs.2018.12.064
}

Lung transplantation has been established worldwide as the final treatment option for severe respiratory failure, saving the lives of many severely ill patients. The 5-year survival is approximately $50 \%$, however, and early mortality exists despite an occurrence rate of less than $10 \% .^{1}$ Ischemiareperfusion injury causes primary graft dysfunction (PGD), a major reason for early death after lung transplantation. Alloantibodies emerge after ischemia-reperfusion injury, and ischemia-reperfusion injury becomes a risk factor for chronic lung allograft dysfunction, a main reason for late death after transplantation. Other issues, such as PGD, acute rejection, donor-specific antibody, chronic lung allograft dysfunction including bronchiolitis obliterans syndrome (BOS), and restrictive allograft syndrome, remain unsolved. Recently, several techniques, including ex vivo lung perfusion and solid-phase antibody screening with identification of single antigens, have been developed and applied widely clinically to solve these complex, multifaceted issues. $^{2}$

Mohanakumar and colleagues ${ }^{3}$ are to be congratulated for their interesting proposal in this issue of the Journal that PGD, acute rejection, donor-specific antibody, and BOS can induce and release exosomes into the circulation, which may play a role in inducing rejection. They have tried to prove this unique idea with multiple publications, including basic research. $^{3-5}$ They have reported observational data sustaining their hypothesis, although they have not proved causality. Nevertheless, their theory could provide physicians and researchers in lung transplantation with meaningful and promising evidence to improve survival after lung transplant. It is hoped that Mohanakumar and colleagues further expand their study from the current preliminary and retrospective investigation $^{3}$ to a more reliable prospective one. For example, the timing of the samples taken in relation to specific events should be described in more detail. Samples other than blood, such as exhaled air, sputum, and bronchoalveolar lavage fluid might be investigated. Moreover, the future. transplantation.

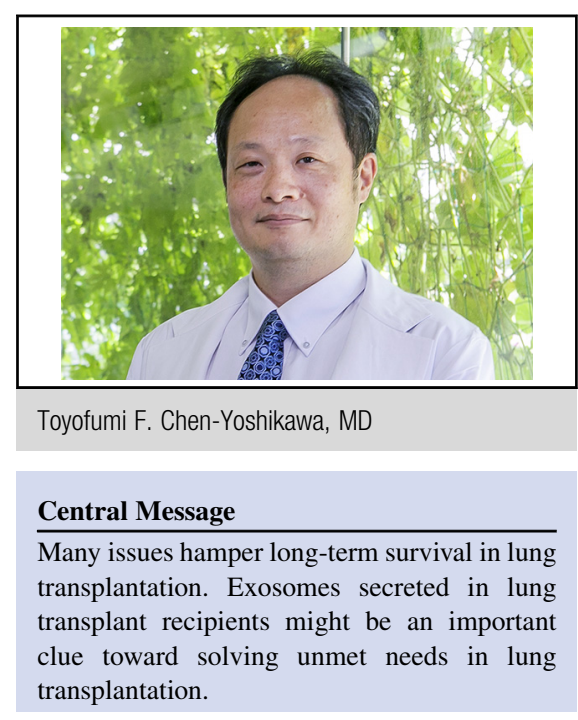

See Article page 2096.

it might be interesting to investigate whether there are linear or other associations with PGD grades other than the binary PGD grade 0 versus grade 3 . With chronic lung allograft dysfunction, restrictive allograft syndrome as well as BOS should be considered, because the restrictive allograft syndrome phenotype is known to be associated with worse survival than the BOS phenotype.

Although recently exosomes have been investigated in the field of transplantation, few reports have described the characteristics of exosomes in body fluids secreted by lung transplant recipients, including blood and bronchoalveolar lavage fluid. It is therefore still uncertain whether exosomes are induced by or the exosomes themselves cause injury in lung transplantation. Further studies are needed in

According to the latest International Society for Heart and Lung Transplantation registry report, the survival curve after lung transplantation between 1999 and 2008 drastically improved in comparison with that between 1990 and 1998, but the survival curve between 2009 and 2016 did not improve in comparison with that between 1999 and 2008. ${ }^{1}$ Further research by Mohanakumar and colleagues ${ }^{3}$ might provide valuable clues to solve these unmet needs and complex issues related to survival after lung

In summary, Mohanakumar and colleagues ${ }^{3}$ showed preliminary observational data indicating that PGD, acute 
rejection, and BOS might be mediated by exosomes, although the mechanism has not been proved.

\section{References}

1. Chambers DC, Cherikh WS, Goldfarb SB, Hayes D Jr, Kucheryavaya AY, Toll AE, et al; International Society for Heart and Lung Transplantation. The International Thoracic Organ Transplant Registry of the International Society for Heart and Lung Transplantation: thirty-fifth adult lung and heart-lung transplant report2018; focus theme: multiorgan transplantation. J Heart Lung Transplant. 2018; 37:1169-83.
2. Chen F, Date H. Update on ischemia-reperfusion injury in lung transplantation. Curr Opin Organ Transplant. 2015;20:515-20.

3. Mohanakumar T, Sharma M, Bansal S, Ravichandran R, Smith MA, Bremner R. A novel mechanism for immune regulation after human lung transplantation. $J$ Thorac Cardiovasc Surg. 2019;157:2096-106.

4. Gunasekaran M, Sharma M, Hachem R, Bremner R, Smith MA, Mohanakumar T. Circulating exosomes with distinct properties during chronic lung allograft rejection. J Immunol. 2018;200:2535-41.

5. Sharma M, Liu W, Perincheri S, Gunasekaran M, Mohanakumar T. Exosomes expressing the self-antigens myosin and vimentin play an important role in syngeneic cardiac transplant rejection induced by antibodies to cardiac myosin. Am J Transplant. 2018;18:1626-35. 